\title{
Sven Fund*
}

\section{Von der Digitalisierung zur Digitalität: Wissenschaftsverlage vor anderen Herausforderungen}

DOI 10.1515/bfp-2017-0020

Zusammenfassung: Wissenschaftsverlage sehen sich einer Vielzahl neuer Herausforderungen gegenüber. Neben einer Änderung des regulativen Umfelds machen es Geschäftsmodelle im Gefolge der Digitalisierung erforderlich, durch interne Maßnahmen und ein geändertes Wettbewerbsverhalten auf Marktherausforderungen zu reagieren.

Schlüsselwörter: Wissenschaftsverlage; Digitalisierung; Geschäftsmodell; Open Access; Verwertungsgesellschaften; Startups

\section{From Digitization to Digitality: Academic Publishers Fa- cing Other Challenges}

Abstract: Academic publishers are confronted with a number of new challenges. Besides significant changes of the regulatory environment, business models have been evolved as a consequence of digitization. They shape publishers' internal reactions as well as their competitive repositioning.

Keywords: Academic publishing; digitization; business model; Open Access; rights and royalties organisations; start-ups

\section{Alles beim Alten?}

„I don't think things will change that much - all the major changes have already happened,“ war sich Derk Haank, CEO des Verlagsriesen Springer Nature, 2009 ganz sicher, nicht ohne einen Ausblick auf das kommende Jahrzehnt zu wagen: „My conclusion is that we are in for a boring decade." ${ }^{1}$ Spätestens aus heutiger Sicht würden die meisten seiner Verlagskollegen ihm wohl widersprechen. Die

1 Allen (2009).

*Kontaktperson: Dr. Sven Fund, sven.fund@fullstopp.com vergangenen 24 Monate haben für Verlage weltweit deutliche Anpassungen des Handlungsrahmens gebracht, die sich in vier Dimensionen analysieren lassen. Neben regulativ-volkswirtschaftlichen Veränderungen sind es wettbewerbliche, kundenseitige und betriebswirtschaftliche Verschiebungen, die das kurzfristige wie das künftige Handeln von Verlagen maßgeblich beeinflussen. Dabei sticht ins Auge, dass diese Dynamik praktisch alle großen Märkte weltweit betrifft, ohne dass die Entwicklungen in einem direkten Zusammenhang $\mathrm{zu}$ einander stehen. Gleichwohl haben sie zusammengenommen teils erheblichen Einfluss auf Wissenschaftsverlage und ihre Geschäftsmodelle.

\section{Mikroökonomische Entwicklung des wissenschaftlichen Publizierens}

Ein Blick in die Geschäftsberichte von börsennotierten Verlagen offenbart, dass trotz gut laufender Konjunktur in den Kernmärkten wissenschaftlichen Publizierens (Europa, Nordamerika und Hauptmärkte Asiens) das Umsatzwachstum der Häuser zum Teil deutlich unter dem der wirtschaftlichen Ergebnisse liegt. So wuchsen die Wissenschaftsgeschäfte von Elsevier und Wiley mit jeweils $2 \%{ }^{2}$ im Umsatz - in Phasen guter konjunktureller Entwicklung eher ernüchternd. Ergebnissteigerungen erreichen die Konzerne also in erster Linie mithilfe von Kostenmaßnahmen. Die Entwicklung erstaunt, deutet doch ein Blick in das Budgetwachstum von akademischen und Corporate Bibliotheken weltweit auf ein deutlich dynamischeres Wachstum. Der Marktforscher Outsell beobachtete für den Zeitraum von 2012 bis 2016 eine jährliche Wachstumsrate im Bereich der wissenschaftlichen Bibliotheken von 2,8 \%, die Ausgaben von Unternehmen für Fachinhalte wuchsen im gleichen Zeitraum sogar um 3,1 \%. ${ }^{3}$ Diese Entwicklung

2 Wiley (2016a) sowie RELX Group (2016).

3 Outsell (2016). 
hat unterschiedliche Gründe, neben Währungsschwankungen ist dies vermutlich insbesondere eine Verschiebung der Ausgaben von Inhalten zu Services rund um Content, die in den vergangenen Jahren nach kursorischen Beobachtungen zum Teil deutlich gestiegen sind.

Getrieben wird das Wachstum der Verlagsunternehmen zu einem großen Teil durch die Konsolidierung des Marktes, die weiter ungebremst voranschreitet. Neben der aufsehenerregenden Verbindung von Springer und dem Wissenschaftsbereich von Macmillan mehrheitlich unter dem Dach der Verlagsgruppe Georg von Holtzbrinck zu Beginn des Jahres 2015 kaufen auch kleinere Marktteilnehmer im deutschsprachigen Raum kontinuierlich zu - mittlerweile insbesondere im digitalen Bereich - und adaptieren so ihr jeweiliges Portfolio. ${ }^{4}$ Dabei verschieben sich die Handlungsmotive der Akteure erheblich: Lag der Anteil digitaler Formate an den Transaktionen 2011 noch bei $42 \%$, so waren es im Jahr 2015 bereits $57 \%$. Verlage kaufen also nicht nur zu, sondern nutzen Akquisitionen aktiv, um ihr Portfolio umzubauen. Und dies gilt auch international: Klassische Buchverlage stehen immer wieder zum Kauf, finden aber nur dann Interessenten, wenn sie für Käufer attraktive Nischen abdecken und idealerweise bereits in hohem Maße digitalisiert sind.

Mit der Insolvenz von Swets im September $2014^{5}$ erlebte die Literaturversorgung von Bibliotheken und Unternehmen eine Krise, die zumindest kurzfristig die Stabilität des etablierten Systems bedrohte und erstmals seit über einer Dekade Verleger (und Bibliothekare) wieder über die Abhängigkeiten bei der Ansprache ihrer Kunden nachdenken ließ. Wenngleich die unternehmerischen Überreste von Swets rasch unter ehemaligen Wettbewerbern des Unternehmens aufgeteilt waren und so Schlimmeres verhindert werden konnte, liefern sich seitdem vor allem EBSCO und ProQuest als weltweit aktive Literaturversorger einen Wettlauf um die Neuausrichtung ihrer Geschäfte. Dabei müssen einerseits die print-basierten Services kontrolliert und ohne gravierende Qualitätseinbußen zurückgefahren werden. Andererseits sind massive Investitionen in den Auf- und Ausbau ihrer Aktivitäten zum Vertrieb digitaler Produkte und von entsprechenden Services erforderlich.

4 Einen guten Überblick über die Mergers \& Acquisitions im deutschsprachigen Raum bietet der jährlich erscheinende Transaktionsmonitor von Bartholomäus (2016).

5 Siehe die Meldung vom Tag der Anmeldung der Insolvenz unter https://www.researchinformation.info/news/analysis-opinion/swets -information-services-bv-declared-bankrupt.

\section{Regulative Veränderungen}

Insbesondere im europäischen Umfeld haben sich für Verlage die Rahmenbedingungen unternehmerischen Handelns in den vergangenen zwei Jahren spürbar verändert, wobei die Veränderungen sowohl auf regionaler, nationalstaatlicher, wie auch auf EU-Ebene stattfinden.

Im regionalen Kontext ist an die zunehmende Anpassung von Promotions- und Habilitationsordnungen in Hochschulen, aber auch Mandate von Forschungseinrichtungen für bestimmte Publikationsweisen zu denken, die Wissenschaftlern zunehmend weniger Freiraum bei der Wahl des Publikationsmodells lassen. ${ }^{6}$ So empfehlen zahlreiche Förderer den von ihnen geförderten Forschern dringend, in Open Access zu publizieren. ${ }^{7}$ Natürlich wird auf dieser wie auch auf anderer Ebene kein Zwang auf Wissenschaftler ausgeübt, in einem spezifischen Modell zu publizieren. Gleichwohl werden finanzielle Mittel in neue Modelle investiert und motivieren so zur Unterstützung dieser Angebote.

Neben der Forscherseite ändern sich auch auf Bibliotheksseite die wirtschaftlichen Handlungsmuster. So werden nach der Meinung praktisch aller Marktbeobachter die derzeit in der Verhandlung befindlichen, bundesweiten DEAL-Lizenzen zum Erwerb von Zeitschrifteninhalten in Deutschland einen erheblichen Einschnitt in die bisherigen Strukturen nach sich ziehen. ${ }^{8}$ Muster für DEAL ist neben den Allianzlizenzen offensichtlich das 2015 getestete Modell der Erwerbungskoordinierung in Sachsen. ${ }^{9}$

Auf nationaler Ebene erfolgreiche Innovationen in der Literaturbeschaffung wurden auch in den Niederlanden und in Österreich getestet. Hier ging es den Initiatoren nicht nur um die Erreichung höherer Volumina und somit besserer Konditionen in den Verhandlungen, sondern insbesondere auch um das Erreichen von Breitenwirkung für ein neues Geschäftsmodell, das zuvor nicht Gegenstand konsortialen Handelns war, die Rede ist von Open Access.

Die Öffnung von Inhalten ist auch auf höchster politischer Ebene innerhalb Europas ein wesentliches Ziel. Gleich drei Kommissare sind innerhalb der EU-Kommission für verschiedene Elemente von Open Science verantwortlich. Freilich geht es dabei nicht um die hehre Wissenschaft und ihre Förderung allein, sondern vor allem um die Erhöhung der wirtschaftlichen Wettbewerbsfähigkeit

\footnotetext{
6 Vgl. etwa das ansonsten wenig innovative Papier des BMBF (2016, 7).

7 Vgl. etwa das Merkblatt Open Access Publizieren der DFG unter http://www.dfg.de/formulare/12_20/12_20_de.pdf.

8 Kellersohn (2016).

9 Golsch und Herrmann (2015).
} 
Europas. So ist denn auch von einem „Digital Single Market“10 die Rede. Neben der Reallokation von Fördergeldern in den Bereichen Open Science und Open Access wird vor allem eine Reform und Harmonisierung des Urheberrechts im EU-Rahmen als probates Mittel gesehen, Markthindernisse aus dem Weg zu räumen und Zugang zu Inhalten zu liberalisieren. ${ }^{11}$

Das Urheberrecht und die Verwertung von Inhalten stehen für deutsche Verleger spätestens seit der Entscheidung des Europäischen Gerichtshofs (EuGH) im Verfahren Hewlett Packard gegen Reprobel im November 2015 fast täglich auf der Agenda, löste die Rechtsprechung doch einen Dominoeffekt auf das Verfahren Vogel gegen die VG Wort aus. Der EuGH hatte in seiner Entscheidung die Beteiligung von Verlagen an den wirtschaftlichen Erlösen aus Lizenzen mit der Begründung verneint, Verlage seien nach Artikel 5 der europäischen Urheberrechtsrichtlinie von 2001 nicht wahrnehmungsberechtigt, es stünden ihnen also keine Zahlungen aus Reprographievergütungen $\mathrm{zu}^{12}$

In seiner Entscheidung im Verfahren gegen die VG Wort folgte der Bundesgerichtshof (BGH) der Entscheidung der europäischen Richter und stürzte vor allem kleinere Verlage in eine existenzbedrohende Situation, deren Dimension aufgrund allerlei offener Umsetzungsfragen derzeit noch nicht geklärt ist. Nach heutiger Einschätzung wird die VG Wort sämtliche Ausschüttungen an Verleger, die für die Jahre 2012 bis 2014 geleistet wurden, zurückfordern müssen - ein Betrag von 99,6 Mio. Euro. ${ }^{13}$

Die wirtschaftlichen Folgen der Forderung sind, obwohl noch gar nicht zur Zahlung fällig, bereits spürbar. So berichtet Walser auf Basis einer Umfrage unter Verlagen von umgehenden Investitionskürzungen, $73 \%$ der Wissenschaftsverlage sahen sich laut Umfrage zu diesem Schritt bereits gezwungen. ${ }^{14}$ Es ist offensichtlich, dass diese verständliche Reaktion in einer ohnehin nicht überinvestierten Branche inmitten der Digitalisierung strukturelle Folgen haben wird. Dies umso mehr, als dass offensichtlich kleine und mittelgroße Verlage mit grundsätzlich eher dünner Eigenkapitalausstattung dem Vernehmen nach härter getroffen sind als größere Marktteilnehmer.

10 Siehe die politische Agenda zum Digital Single Market unter https://ec.europa.eu/priorities/digital-single-market_en.

11 European Commission (2016).

12 Vgl. Poll (2015).

13 Hierbei handelt es sich allein um die Rückforderung der VG Wort an Verlage, hinzu kommen analoge Forderungen anderer Verwertungsgesellschaften wie der VG Bild Kunst, die teilweise ebenfalls Verlage betreffen. Vgl. Sprang (2016).

14 Walser $(2016,2)$.

\section{Wettbewerbliche Veränderungen}

Die Konsolidierung der Verlagsbranche im produzierenden wie im vertreibenden Bereich schreitet weiter voran. Waren dafür früher insbesondere Skaleneffekte in den klassischen Herstellungsprozessen verantwortlich, sind dies heute Investitionen in Technologien und Knowhow bei Mitarbeitern, das in kurzer Zeit aufgebaut werden muss. Neben dem klassischen Kauf von Unternehmen oder Unternehmensanteilen gewinnen offensichtlich Kooperationsmodelle an Bedeutung, etwa im Rahmen der JurisAllianz, in der sich rechtswissenschaftliche Verlage zur Publikation von Inhalten in komplexen Datenbankenstrukturen zusammengeschlossen haben. Auch Modelle wie das des Verlags De Gruyter, der seit Jahren seine Marktposition durch strategische Partnerschaften bei der Verbreitung digitaler Inhalte ausbaut, finden zunehmend Nachahmer. ${ }^{15}$

Verlage treiben also die Konsolidierung voran, um ein sich abschwächendes Marktwachstum durch Zukäufe zu kompensieren und Skalenvorteile in Kosten und Investitionsbedarf zu erreichen. Daneben suchen gerade große Player kreative Wege, ihren Anteil an der Wertschöpfung in Verlagsprozessen zu erhöhen - etwa Wiley mit dem im Sommer 2016 angekündigten Kauf des Hosting-Dienstleisters Atypon. ${ }^{16}$ Diese Akquisition folgt in ihrer Logik der durchaus oszillierenden - Suche nach Service-Modellen für wissenschaftliche Gesellschafter und kleine Verlage. Diese erkennen angesichts der wachsenden Globalisierung und der durch die Fragmentierung der Angebotsformen steigenden Komplexität ihres Geschäfts zunehmend, dass ihre Mittel zu begrenzt sind, um international mitspielen zu können. Zugleich besteht offensichtlich bei Verlagen wie Wiley und Oxford University Press (OUP) ein Bedarf, durch das Anbieten weiterer Services ihre Strukturkosten im Vertrieb und in anderen Bereichen zu decken und zugleich Partner auf Gesellschaftsseite durch umfangreichere Problemlösungen nachhaltig an sich zu binden.

Es lassen sich dabei zwei Typen von Integration unterscheiden. Während beispielsweise OUP auf Volumenwachstum seiner Inhalte durch die Integration von Verlagspartnern setzt, streben Elsevier und die Nature Publishing Group die Integration von Partnern in neue Geschäftsmodelle an. ${ }^{17}$

15 In Deutschland arbeitet Nomos nach einem vergleichbaren Modell, als international relevanter Wettbewerber ist Oxford University Press zu nennen.

16 Vgl. Wiley (2016b).

17 Hier ist im Fall von Elsevier an den Kauf und die Integration von Mendeley zu denken. 
Getrieben wird die Konsolidierung auf Seiten der Verlage durch eine Abschwächung der Wachstumsdynamik in „neuen“ Märkten Asiens, Lateinamerikas und des Nahen Ostens, die in den letzten Jahren erhebliches Wachstum, insbesondere bei digitalen Inhalten, gezeigt und so Gewinn- und Verlustrechnungen der Unternehmen aufpoliert hatten.

\section{Kundenseitige Veränderungen}

Neben der abnehmenden Bedeutung neuer Märkte als Wachstumstreiber für etablierte Wissenschaftsverlage sorgen auch andere Faktoren im Gefolge der Digitalisierung für Veränderungsdruck. Noch ist die Richtung, aus denen diese Veränderung kommt, schwierig zu bewerten. So haben im (digitalen) Buchgeschäft, insbesondere in Nordamerika, Demand-Driven-Acquisition-Modelle (DDA) in einem Maß an Bedeutung gewonnen, dass sie das Erwerbungsverhalten von Bibliotheken nachhaltig beeinflussen und $\mathrm{zu}$ teilweise tiefgreifenden Budgetverschiebungen führen. ${ }^{18}$ Diese Art von Angeboten, bei denen Bibliotheken Metadaten in ihre Katalog einspielen, der eigentliche Kauf der Inhalte aber erst in der konkreten Nutzungssituation geschieht, führen gerade bei Kunden, bei denen Bestandsaufbau klassischer Art nicht an erster Stelle steht, zu einer deutlichen Umschichtung von Mitteln - mit unklaren Folgen für die Einrichtungen selbst, wie Beobachter betonen.

Parallel zum Aufkommen von DDA bei im Wesentlichen stabiler Haushaltslage entwickeln Bibliothekare ein wachsendes Bedürfnis, Entscheidungen stärker quantitativ zu treffen. Diese Ausschöpfung digitaler Möglichkeiten hat zur Folge, dass die Nutzung von Inhalten in Bibliotheken massiv an Bedeutung für künftige Erwerbungen gewonnen hat - ein Trend, der sich seit ein paar Jahren abzeichnet. Ausschlaggebend scheint dabei, dass quantitative Entscheidungen offensichtlich verstärkt auch dort auf konkrete Käufe durchschlagen, wo die Angebotsstrukturen dies bisher vermeiden konnten, insbesondere also im Big Deal. Nach Aussagen von Bibliothekaren werden auch diese Pakete großer Anbieter bei schlechter Nutzung eines Teils des Portfolios konsequenter abbestellt als früher.

Verlage sehen sich mit neuen Anforderungen in Bereichen konfrontiert, für die sie Lösungen entwickeln könnten, etwa im Data Publishing. Allerdings konnten in den vergangenen Jahren bisher keine nachhaltigen Monetarisierungsmodelle für dieses noch junge Feld entwickelt

18 Vgl. etwa Zeoli (2015). werden, so dass Anbieter derzeit von der Realisierung dieser Option Abstand nehmen.

Der Eintritt neuer Wettbewerber ist durch die Digitalisierung des wissenschaftlichen Publizierens in einem Maße begünstigt worden, wie die Branche es vorher nicht kannte. Nun sind die Markteintrittsbarrieren im Verlagswesen traditionell eher niedrig gewesen, sie haben sich jedoch in den letzten beiden Dekaden eher zugunsten der Etablierten erhöht. Gerade Open Access (OA), ursprünglich angetreten als politischer Gegenentwurf zur wachsenden Marktmacht der Großen in der Zeitschriftenkrise der 1990er-Jahre, hat mit diesem Trend zumindest zum Teil gebrochen. Zwar haben die Marktführer auch im OA längst große Marktanteile unter ihre Kontrolle gebracht - die Liberalisierung des Vertriebs von Inhalten haben sie jedoch nicht aufgehalten, im Gegenteil. Und Plattformen wie Academia.edu, die mittlerweile über 42 Millionen registrierte Nutzer zählt, haben sich als nicht-kommerzielle Alternative zur Verbreitung wissenschaftlicher Inhalte fest etabliert. ${ }^{19}$ Aus Autorensicht scheinen diese Modelle interessant, weil sie Wissenschaftlern die Möglichkeit geben, Zielgruppen für die Distribution ihrer Arbeit zu definieren und sehr detailliert Aufschluss über Nutzung und Zitierung zu erhalten - ein Angebot, dass praktisch kein Wissenschaftsverlag in dieser Form anbieten kann.

Die Kehrseite der Digitalisierung erlebten Wissenschaftsverlage Anfang 2016. Die junge Wissenschaftlerin Alexandra Elbakyan hatte 2015 damit begonnen, derzeit über 58 Millionen ${ }^{20}$ Dokumente unter einer gemeinsamen Suchoberfläche und für Nutzer kostenlos zu sammeln. ${ }^{21}$ Ohne Frage handelt es sich um einen massiven Verstoß gegen Urheber- und vor allem Verbreitungsrechte, die die Unterstützer der Seite als legitime Demokratisierung verbrämten, während andere Beobachter den „Napster Moment“ der Verlagsindustrie gekommen sahen. ${ }^{22}$ Der Vergleich ist insofern zutreffend, als dass John Bohannon mittlerweile zeigen konnte, wie international die Nutzung von Sci-Hub durch Wissenschaftler ist. ${ }^{23}$ Er hat damit die Theorie widerlegt, die Nutzung illegal zur Verfügung gestellter Inhalte sei (auch aufgrund der umfangreichen Lizenzierung von Inhalten durch wohlhabende Institutionen) in erster Linie eine Randerscheinung und wirtschaftlich für Verlage nicht von Belang.

19 Vgl. https://www.academia.edu.

20 Vgl. https://scihub22266oqcxt.onion.link.

$21 \mathrm{Zu}$ Struktur und Funktionsweise von Sci-Hub vgl. https://scholar lykitchen.sspnet.org/2016/02/25/sci-hub-how-does-it-work/.

22 Siehe stellvertretend Coldewey (2016).

23 Bohannon (2016). 


\section{Betriebswirtschaftliche Kon- sequenzen}

Verlage sind, nicht nur in der eigenen Wahrnehmung, mit einer Vielzahl recht tiefgreifender Veränderungen konfrontiert, die ihr unternehmerisches Handeln beeinflussen. Dabei ist offensichtlich, dass der größere Anteil der Faktoren durch die Digitalisierung von Produkten und eben zunehmend auch von Prozessen getrieben wird. Während dies nicht direkt für die Veränderungen des regulativen Umfelds zutrifft, sei die These gewagt, dass die Branche durch ihr Vorgehen in der frühen Phase der Digitalisierung einige der Effekte selbst verursacht hat, unter denen sie jetzt leidet. Dieser Befund bezieht sich insbesondere auf die Taktik, bereits in gedruckter Form vorhandene Informationen in großen Retrodigitalisierungsprojekten nochmals zum Kauf anzubieten und so erhebliche Mittel in neue Medien $\mathrm{zu}$ investieren, die jedoch nur selten Geschäftsmodelle anbieten, die dem Einmal-Charakter dieser Vertriebserfolge Rechnung getragen hätten. Offensichtlich fand dieses Vorgehen ja auch auf Kundenseite und durch die Nutzer breite Unterstützung - einer Zukunftsperspektive der Geschäftsmodelle von Verlagen ist es hingegen nicht zuträglich.

Der Trend zu einer Spaltung des Marktes im wissenschaftlichen Publizieren hat sich in der vergangenen Dekade beschleunigt. Kapitalstarken und risikotoleranten Unternehmen ist es so gelungen, einen wachsenden Anteil der verfügbaren Kundenbudgets auf sich zu vereinigen, und das in praktisch allen Geschäftsmodellen. Es kann nur gemutmaßt werden, welcher der beiden Faktoren, Kapital und Risikotoleranz, eine größere Rolle spielt. Faktoren wie die Kommoditisierung der Zulieferfunktionen (etwa in der Herstellung), Erleichterungen im Welthandel und eine zunehmende Internationalisierung der Wissenschaften haben Ersparnisse möglich gemacht, die großen wie kleinen Anbietern gleichermaßen zugute kommen. Gleichzeitig ist zu beobachten, dass Innovationen - häufig aus dem Bereich der Nutzer getrieben - von großen Marktteilnehmern früh erkannt und konsequenter in Geschäftsmodelle umgesetzt werden. Beispielhaft hierfür ist der kreative und kommerziell sehr erfolgreiche Umgang von Springer mit Open Access. Im Ergebnis dominieren die Großen der Branche auch neue Geschäftsmodelle und bauen so ihre Marktposition aktiv aus.

Parallel hierzu haben zahlreiche Unternehmen schon vor Jahren damit begonnen, an den internen Stellschrauben zur Senkung ihrer Kosten zu arbeiten, um in weniger wachstumsstarken Zeiten trotzdem ihre Profitabilität auszubauen. Großflächiges Outsourcing aller möglichen
Dienstleistungen hat dabei über die Jahre zu einer Veränderung des Wertschöpfungsmodells geführt, in dem die vertriebliche Dimension zeitweise deutlich an Gewicht gewonnen hat. So wuchsen auch in Zeiten restriktiver Personalkostensteigerungen die Vertriebsabteilungen in vielen Verlagen und es wurden Mittel in die Hand genommen, um Distributionsplattformen zu stärken.

\section{Von der Digitalisierung zur Digitalität - was erwartet Verlage?}

Digitale Geschäftsmodelle ziehen ihr Wachstum in etablierten Industrien sehr häufig aus DisintermediatisierungsEffekten. Wo früher, wie in der Musikindustrie, massive Vertriebslogistik nötig war, um bei Veröffentlichung einer CD Tonträger praktisch gleichzeitig in die Musikläden der Welt zu befördern, gelingt dies heute auf digitalem Wege völlig anders. Der Einwand, digitale Vertriebskanäle seien auch von wenigen sehr großen Marktteilnehmern dominiert und der Markt sei lediglich umverteilt worden, trifft allerdings nur zum Teil zu: Das Umsatzvolumen der Musikindustrie weltweit sank zwischen dem Jahr 2000 und 2015 um 52,5\%.24

Verlage sind, das zeigt die hohe Popularität von Modellen wie Acadmia.edu und illegaler Angebote wie SciHub, massiv durch diese Disintermediatisierung bedroht. Ihre Reaktionsweisen sind umso eingeschränkter, als heute nicht nur die technischen Möglichkeiten zur Verfügung stehen, auf den Middle-Man-Verlag zu verzichten. Vor allem sind zahlreiche Modelle mit der expliziten Zielsetzung entwickelt worden, diesen Schritt zu machen und die hohen Renditen der Branche - abgeleitet aus den Ausnahmewerten der Marktführer - gleichsam zu resozialisieren.

Ein closed loop zwischen den Erzeugern und den Rezipienten wissenschaftlicher Inhalte funktioniert nach heutigen Erkenntnissen nur partiell, womit sich auch in der Zukunft eine - gewandelte - Rolle von Verlagen im Kommunikationsprozess rechtfertigen lässt.

Schon heute reagieren etablierte Unternehmen marktseitig mit einer Ausrichtung auf diese neuen Realitäten. Zahlreiche Services unterstützen Unternehmen dabei, eine höhere Nutzerorientierung und bessere Transparenz des

24 https://de.statista.com/statistik/daten/studie/182361/umfrage/w eltweiter-umsatz-der-musikindustrie-seit-1997/. 
Publikationsprozesses zu schaffen. Anbieter wie Kudos, PaperHive oder Publons ergänzen bestehende Angebote von Verlagen. ${ }^{25}$ Kudos etwa ermöglicht Autoren, ihre Artikel selber effizient in sozialen Medien zu bewerben. PaperHive treibt durch Interaktion von Wissenschaftlern rund um einen Artikel die Nutzung wissenschaftlicher Inhalte voran - angesichts der Aufwertung von Nutzung zur Zweitwährung im Handeln zwischen Bibliotheken und Verlagen ein zentrales Desiderat. Und Publons gibt Forschern, die sich aktiv am Peer-Review-Prozess beteiligen, die Möglichkeit, diese praktisch unsichtbare und nicht bewertbare Tätigkeit in immer stärker auf basisanalytischer Verfahren stattfindenden Berufungsverfahren als Teil ihres akademischen Schaffens zu identifizieren.

\section{Neue Flexibilität durch Startups?}

Wissenschaftsverlage haben über die vergangenen Dekaden eine bemerkenswerte Resistenz gegenüber Veränderungen in ihren Kernprozessen gezeigt - und damit erheblich zur Stabilität der wissenschaftlichen Informationsversorgung beigetragen. Natürlich diente die geringe Veränderungsneigung gerade auch ihnen selbst. Sie half, Investitionen zu senken und Profite zu steigern. Andere Teile des Ökosystems rund um Verlage - Satzbetriebe, Drucker, Händler und nicht zuletzt die Wissenschaften selbst - mussten im gleichen Zeitraum deutlich höhere Anpassungsleistungen erbringen, um im Geschäft zu bleiben. Nur Bibliotheken scheinen es Verlagen gleich zu tun und eher zurückhaltende Innovatoren zu sein.

Startups als eine neue Form unternehmerischen Handelns spielen im wissenschaftlichen Publizieren eine zunehmend wichtigere Rolle, kaum eine Fachkonferenz kommt noch ohne sie aus. Häufig angetreten als Spezialisten mit der Zielsetzung, ein spezifisches Problem zu lösen, hat die Verlagsbranche früh, wenn auch unsystematisch mit diesen neuen Akteuren kooperiert. Prominentestes Beispiel ist der Aufbau der Digital-Science-Gruppe innerhalb des Holtzbrinck-Konzerns, mit dem es über die vergangenen Jahre gelungen ist, innovative Ansätze finanziell und inhaltlich zu fördern, ohne ihnen das disruptive Potenzial zu nehmen. Neben den Marktführern wie Elsevier, die bisher eher informelle Organisationsformen zur frühen Bindung von Startups an sich nutzen, gehen mitt-

$25 \mathrm{Zu}$ den Angeboten der Startups und ihrer Arbeitsweise vgl. https://www.growkudos.com, https://paperhive.org und http://prw. publons.com. lerweile auch kleinere Häuser den Weg externer Innovation. Der Verlagsberater David Worlock spricht in diesem Kontext zutreffend von regenerativer Innovation, die einer Wiederaufladung der traditionellen Kerngeschäfte von Verlagen dient.

\section{Ausblick}

Wissenschaftsverlage geraten mit wachsender Intensität unter Anpassungsdruck. Qualitativ ändert dieser sich insofern, als dass Veränderung nicht mehr nur umsatzseitig und experimentell stattfinden kann, sondern zusehends in Kernprozesse der Unternehmen und in deren Selbstverständnis eingreifen muss. Im deutschen Kontext ist dafür insbesondere die als bedrohlich empfundene Entscheidung des BGH, die effektiv zu einer Zerschlagung der Verwertungsgesellschaften in ihrer bisherigen Form geführt hat, als Wendepunkt zu betrachten. Dabei geht es nicht in erster Linie um die auf Verlage zukommenden Rückforderungen, sondern vor allem um die Wahrnehmung, Verlage würden am Prozess der Wissenserzeugung und -distribution keinen signifikanten Anteil haben.

Verlage befinden sich - wie auch Bibliotheken - in einem Umfeld, das gleichsam den Aggregatzustand von der Digitalisierung zur Digitalität ändert. Es ist nicht mehr ausreichend, Produkte (auch) in digitaler Form anzubieten und in der Folge die Prozesse entsprechend umzustellen. Vielmehr zeigen Geschäftsmodelle wie Demand bzw. Patron Driven Acquisition, dass der Übergang vom klassischen Verkauf zur Lizensierung bzw. der zeitweisen Nutzung eingeleitet ist. Damit verbunden ist die Notwendigkeit spezifischer Reaktionen in Verlagen, etwa in der Kalkulation von Produkten. Nie war die Notwendigkeit von Standardisierung so erforderlich wie jetzt, wollen Verlage den vollen Nutzen der Digitalität an die Nutzer weitergeben können.

Letztlich sind die Gewichte in der Wissenschaftskommunikation ins Rutschen geraten, weg von der reinen Bereitstellung von Inhalten hin zum Angebot umfangreicher Serviceleistungen rund um Content. Befeuert wird diese Entwicklung durch mittlerweile schier unübersichtliche Mengen an Digitalisaten, die zur Verfügung stehen und sinnvoll nutzbar gemacht werden müssen.

Neue Formen des Wirtschaftens werden nötig, und Verlage tun gut daran, einen Blick in andere Branchen zu werfen. Es ist dringend erforderlich, die Erzeuger und Nutzer von Inhalten verstärkt in den Fokus zu nehmen, soll die Abstimmung mit den Füßen gegen klassische Angebote von Verlagen gestoppt werden. Innovative Modelle, wie Startups sie bieten, sind dabei für etablierte Player sicher 
ein gangbarer Weg, Innovation zu beschleunigen und zugleich Risiken zu begrenzen. ${ }^{26}$

\section{Literaturverzeichnis}

Allen, Katherine (2009): Springer Is Not for Sale, Says CEO. Verfügbar unter http://newsbreaks.infotoday.com/NewsBreaks/SpringerIs-Not-for-Sale-Says-CEO-53277.asp, veröffentlicht am: 02.04.2009.

Bartholomäus, Axel (2016): Transaktionsmonitor Verlagswesen 2015. Synopsis der M\&A-Aktivitäten der deutschen Printmedien und Analyse der Entwicklung von 2011 bis 2015. Verfügbar unter http://www.ba-cie.de/transaktionsmonitor.html.

BMBF (2016): Open Access in Deutschland. Verfügbar unter https:// www.bmbf.de/pub/Open_Access_in_Deutschland.pdf.

Bohannon, John (2016): Who is downloading pirated papers? Everyone. In: Science, 28.04.2016. Verfügbar unter DOI: 10.1126/science.aaf5664.

Coldewey, Devin (2016): Sci-Hub is providing science publishers with their Napster moment. In: Techcrunch, (29.04.2016). Verfügbar unter https://techcrunch.com/2016/04/29/sci-hub-is-providing-science-publishers-with-their-napster-moment/, zugegriffen am: 20.09.2016.

European Commission (2016): State of the Union 2016: Commission proposes modern EU copyright rules for European culture to flourish and circulate. European Commission. Verfügbar unter http://europa.eu/rapid/press-release_IP-16-3010_en.htm, veröffentlicht am: 14.09.2016, zugegriffen am: 20.09.2016.

Golsch, Michael; Herrmann, Jana (2015): Erwerbungskoordinierung im Sachsenkonsortium: Eine Zwischenbilanz. In: BIS - Das Ma gazin der Bibliotheken in Sachsen, 82 (2), 82-85. Verfügbar unter urn:nbn:de:bsz:14-qucosa-173525.

Kellersohn, Antje (2016): DEAL - Bundesweite Lizenzierung von Angeboten großer Wissenschaftsverlage. Verfügbar unter http:// www.bibliotheksverband.de/fileadmin/user_upload/Kommissionen/20160429_DEAL_erwerbungskommission.pdf.

Outsell (2016): 2016 Library Market. Size, Share, Forecast, and Trends. May 5, 2016. Verfügbar unter https://www.outsellinc.co $\mathrm{m} /$ search/d7entity/107130.

Poll, Günter (2015): Verwertungsgemeinschaften dürfen Verleger nicht mehr beteiligen (Legal Tribune Online: 13.11.2015). Verfügbar unter http://www.lto.de/recht/hintergruende/h/eugh-urteilc572-13-verwertungsgesellschaften-verleger-vg-wort-gema, zugegriffen am: 20.09.2016.

RELX Group (2016): Annual Reports and Financial Statements 2015. Verfügbar unter http://www.relx.com/investorcentre/reports\% 202007/Documents/2015/relxgroup_ar_2015.pdf.

Sprang, Christian (2016): Rückforderungen von Verwertungsgesellschaften - was können Verlage tun? (Börsenblatt Online: 06.09.2016). Verfügbar unter https://www.boersenblatt.net/ar tikel-analyse_von_boersenvereinsjustiziar_christian_sprang_ teil_2_.1160882.html, veröffentlicht am: 2016, zugegriffen am: 20.09.2016.

26 Ein Beispiel für potenziell transformative Startups ist Mendeley, das die Zusammenarbeit in Forschergruppen neu organisieren hilft, vgl. https://www.mendeley.com.
Walser, Aljoscha (2016): Folgen des BGH-Urteils zu VG Wort-Ausschüttungen für Verlage. Bad Vilbel: Narses.

Wiley (2016a): Fourth Quarter and Fiscal 2016 Earnings Report, June 14, 2016. Verfügbar unter http://eu.wiley.com/WileyCDA/PressRelease/pressReleaseld-126182.html.

Wiley (2016b): Wiley Signs Definitive Agreement to Acquire Atypon. Verfügbar unter http://eu.wiley.com/WileyCDA/PressRelease/p ressReleaseld-127702.html, zugegriffen am: 20.09.2016.

Zeoli, Michael (2015): Academic Libraries and the Scholarly Book Marketplace: Death by 1,000 [Paper]Cuts? In: Against the Grain, 27 (5), 14-16.

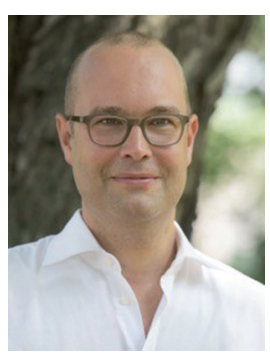

\section{Dr. Sven Fund}

Geschäftsführer Fullstopp GmbH

Wartburgstraße 25A

D-10825 Berlin

sven.fund@fullstopp.com 